\title{
Aggravation of Inappropriate Luteinizing Hormone Secretion by Bromocriptine in Polycystic Ovary Syndrome with Elevated Serum Dehydroepiandrosterone Sulfate
}

\author{
Kenji TAKAKURA, SHunzo TAII, YoshiYuki IHARA, IchIRo TAKAI \\ AND TAKAHIDE MORI \\ Department of Gynecology and Obstetrics, Kyoto University Faculty of \\ Medicine, Kyoto 606, Japan
}

\begin{abstract}
Ten clomiphene-resistant, normoprolactinemic women with polycystic ovary syndrome (PCOS) were treated by continuous and increasing administration of bromocriptine $(\mathrm{Brc})$, and changes in hormonal profiles as well as therapeutic efficacy were examined. Ovulation was restored in four patients (responders), and two of them became pregnant, whereas the other six patients did not ovulate with Brc alone (non-responders). Endocrine analyses revealed distinct differences between responders and non-responders: 1) pretreatment serum levels of dehydroepiandrosterone sulfate (DHAS) in non-responders were significantly higher than those in responders: 2) exaggerated $\mathrm{LH}$ secretion was definitely aggravated with Brc therapy in non-responders, but unchanged or slightly reduced in responders: 3) basal PRL secretions showed a marked reduction in both groups, whereas this response to TRH in responders decreased more markedly than in non-responders with the therapy.

It is concluded that low DHAS group patients of PCOS are likely to respond to $\mathrm{Brc}$, whereas high DHAS group patients appear contraindicated for this treatment due to its aggravating effect on LH secretion.
\end{abstract}

Although the precise etiology of polycystic ovary syndrome (PCOS) is still to be elucidated, recent studies have suggested that hypothalamic dopamine (DA) deficiency may be involved in causing inappropriate gonadotropin secretion characteristic of PCOS (Quigley et al., 1981, Cumming et al., 1984). Based upon this

Received November 24, 1988

Address all correspondence and requests for reprints to: Dr. KENJI TAKAKURA, Department of Gynecology and Obstetrics, Kyoto University Faculty of Medicine, Shogoin Kawaharacho 54, Sakyo-ku, Kyoto 606, Japan. hypothesis, normalization of hypothalamic dopamine turnover in PCOS patients has been attempted by several investigators using a potent dopamine agonist, bromocriptine (Brc). However, the effectiveness of this drug in PCOS patients through improving inappropriate $\mathrm{LH}$ secretion has remained controversial, since some investigators have successfully induced ovulation and corrected excess secretion of $\mathrm{LH}$ and androgens with this treatment (Spruce et al., 1984, Falaschi et al., 1986), while others have failed to achieve such an effect (Buvat et al., 1986). In this study, on the basis 
that the endocrine status of PCOS patients may not be homologous (Yen 1980, Coney 1984, Futterweit 1984) especially in terms of adrenal function (Lachelin et al., 1979, Loughlin et al., 1986), we performed longterm Brc therapy in clomiphene-resistant PCOS patients in order to clarify the changes in endocrine profiles as well as clinical efficacy associated with Brc administration with the aim of obtaining some predictable indicators of clinical efficacy of this drug.

\section{Materials and Methods}

\section{Patient Selection and Treatment Protocol}

Ten clomiphene-resistant anovulatory patients, aged from 25 to 34 , visiting the Infertility Clinic at the Department of Gynecology and Obstetrics of nyoto Univerity Hospital were diagnosed as PCOS by clinical symptoms and laboratory evidence of hyperandrogenemia and hypersecretion of $\mathrm{LH}$ as essentially described by Yen (1980). These patients did not ovulate even after clomiphene treatment $(100 \mathrm{mg} /$ day for 5 days starting on the 5 th day of the induced menstrual cycle) for at least 3 consecutive cycles. All of these patients were amenorrheic with ultrasonographically confirmed polycystic ovaries (Parisi et al., 1984, Orsini et al., 1985). Hirsutism is rare in Japanese women with PCOS (Kurachi et al., 1971, Aono et al., 1977), and none of our patients were hirsutes. Hypersecretion of $\mathrm{LH}$ was determined either by elevated basal $\mathrm{LH}(>23 \mathrm{mIU} / \mathrm{ml})$ or excess response to $\mathrm{GnRH}(>81 \mathrm{mIU} / \mathrm{ml})$, as described below. Hyperandrogenemia was evaluated from elevated serum levels of 44 -androstenedione (ASD) $(>1.4$ $\mathrm{ng} / \mathrm{ml})$ and/or testosterone $(\mathrm{T})(>0.40 \mathrm{ng} / \mathrm{ml})$. These values were set as the mean $+3 / 2 \mathrm{SD}$ of the measurements in our laboratories for the control subjects. Patients with hyperprolactinemic PCOS (basal PRL $>25 \mathrm{ng} / \mathrm{ml}$ ) were excluded to avoid confusion, because the effect of $\mathrm{Brc}$ on PRL secretion and accompanying changes in $\mathrm{LH}$ and androgens could induce ovulation. Eight informed volunteers with regular menstrual cycles served as control subjects.

The patients received Brc continuously at three different dose levels of 1.25, 2.5, and
$5 \mathrm{mg}$ daily for 4 weeks for each dosage. Starting from the lowest dose, effective dosage for inducing ovulation was maintained thereafter. If ovulation was not iduced by the highest dose of Brc alone, $100 \mathrm{mg}$ clomiphene daily was combined with this drug from day 5 of the menstrual cycle for 5 days in the subsequent cycles. The patients who demonstrated restored ovulatory cycles with Brc were referred to as responders, and those who remained anovulatory with this drug alone as non-responders. Ovulation was evaluated by ultrasonographic observation of follicles and serum levels of progesterone.

\section{Blood Sampling and Hormone Measurements}

To avoid the effects of daily fluctuations and circadian variations in each patient (Rebar et al., 1976), blood samples for the measurements of basal hormonal levels were taken from $9: 30$ to $10: 30 \mathrm{~A}$. M. as often as possible with 6.7 mean sampling times before Brc administration and 3.9 times in the last treatment cycle during the Brc treatment for each patient, and mean basal levels were calculated. All samplings were performed soon after induced withdrawal bleeding or early in each treatment cycle.

A double loading test with simultaneous intravenous injection of $100 \mu \mathrm{g} \mathrm{GnRH}$ and $250 \mu \mathrm{g}$ TRH (Tanabe Pharmaceuticals Co. Ltd., Osaka, Japan) was performed before treatment and at the end of every treatment cycle as previously described (Lin et al., 1986). The net increase in $\mathrm{LH}$ and FSH for the first 30 minutes after loading GnRH was defined as $\triangle \mathrm{LH}$ and $\triangle \mathrm{FSH}$, respectively. Likewise, $\triangle \mathrm{PRL}$ was designated as the net increase in PRL for the first 15 minutes after the injection of TRH. In 6 patients, in whom the pulsatile secretion pattern of $\mathrm{LH}$, FSH, and PRL was studied, blood sampling was perfomed every 15 minutes for 3 to 5 hours before, during, and at the end of the treatment.

Serum levels of LH, FSH, and PRL and those of progesterone $(P)$, estradiol $\left(E_{2}\right)$, and $T$ were measured by RIA using commercial kits purchased from Daiichi Radioisotope Laboratories, Ltd. (Tokyo, Japan) and CIS (the Commissariat A L'Energie Atomique, France), respectively (Lin et al., 1986). Dehydroepiandrosterone sulfate (DHAS), dehydroepiandrosterone (DHA), ASD, and estrone $\left(\mathrm{E}_{1}\right)$ were measured by the methods reported by Kokubu 
et al. (1978) and Ohara et al. (1987).

The intrassay coefficients of variation were $5.5 \%$ for $\mathrm{LH}$, $6.5 \%$ for $\mathrm{FSH}, 6.8 \%$ for PRL, $5.0 \%$ for $\mathrm{E}_{2}, 6.5 \%$ for $\mathrm{E}_{1}, 5.7 \%$ for $\mathrm{P}, 6.7 \%$ for $\mathrm{T}, 6.2 \%$ for DHAS, $5.4 \%$ for DHA, and $6.5 \%$ for ASD, respectively. The interassay coefficients of variation were $10.7 \%$ for $\mathrm{LH}$, $10.5 \%$ for $\mathrm{FSH}, 9.5 \%$ for PRL, $5.7 \%$ for $\mathrm{E}_{2}, 11.0 \%$ for $\mathrm{E}_{1}, 11.5 \%$ for $\mathrm{P}, 9.9 \%$ for $\mathrm{T}, 8.9 \%$ for DHAS, $10.2 \%$ for DHA, and 8.7\% for ASD, respectively.

Statistical comparison was performed using analysis of variance, followed by Duncan's multiple range test.

\section{Results}

Four out of ten patients demonstrated restored ovulatory cycles with Brc therapy (responders), and two of them became pregnant. After the initiation of this therapy, the first ovulations occurred in 0.6-2.6 months with an average period of 1.4 months, in the course of treatment periods which ranged from 1.5 to 8.0 months, with an average of 4.4 months. The other six patients did not respond to Brc and remained anovulatory (non-responders) during treatment periods ranging from 3 to 6 months with an average of 3.9 months. However, when clomiphene was added to Brc treatment in non-responders, two of them responded.

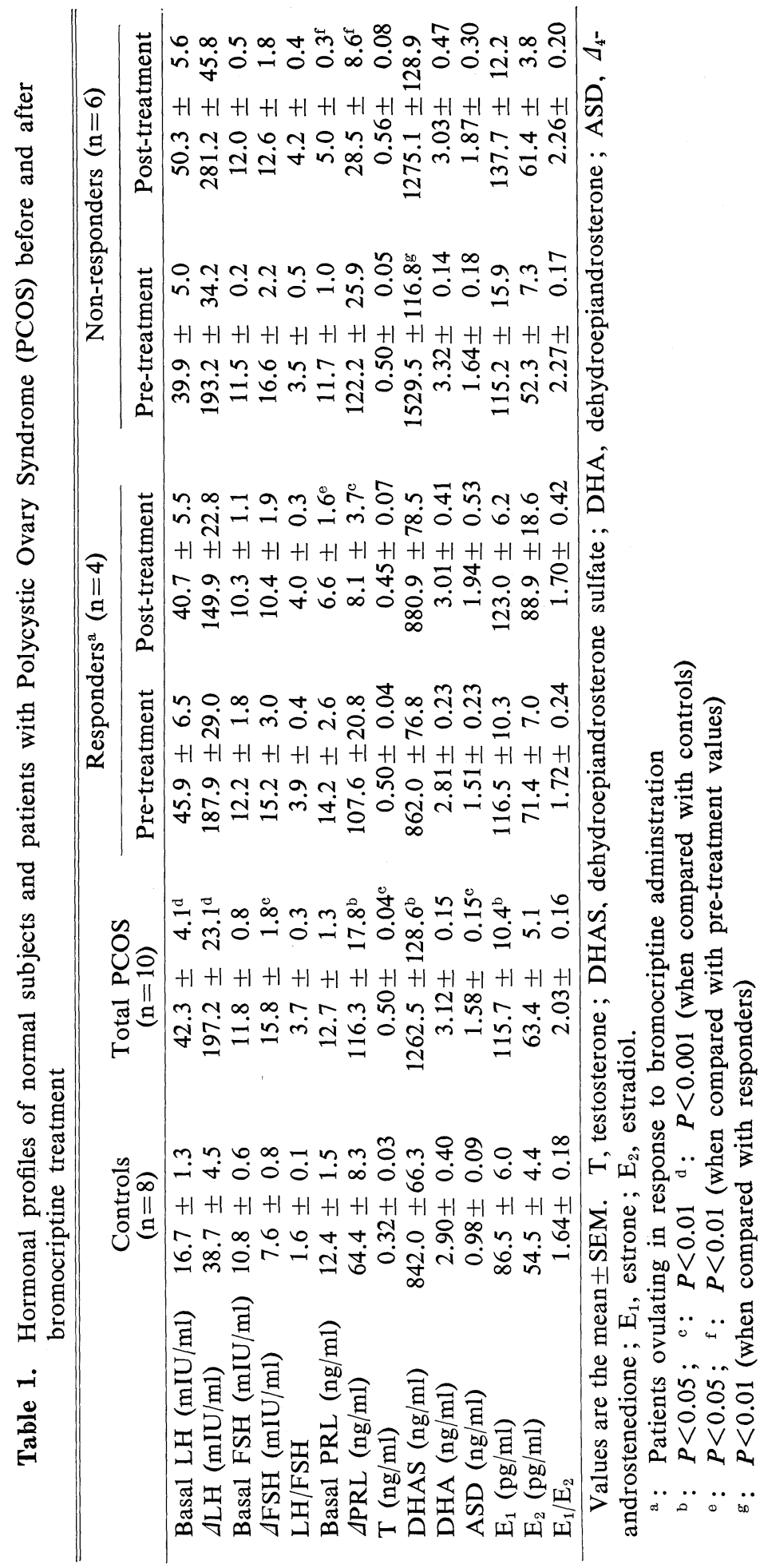


The hormonnal profiles of responders and non-responders before and after $\mathrm{Brc}$ treatment are shown in Table 1 together with \%changes (comparison of posttreatment values with pre-treatment values) in Fig. 1. Among pretreatment values of the hormones measured, only DHAS in non-responders $(1529.5 \pm 116.8 \mathrm{ng} / \mathrm{ml})$ was significantly higher $(P<0.01)$ than that of responders $(862.0 \pm 76.8 \mathrm{ng} / \mathrm{ml})$. Though not significant, DHAS tended to decrease after Brc administration in non-responders only. Basal LH concentrations tended to decrease in responders with Brc administration, in contrast to an increasing tendency in non-responders. This discrepancy became clearer when the \% decrease in responders $(89.7 \pm$ $5.7 \%$ ) was compared to the $\%$ increase in non-responders $(130.7 \pm 15.2 \%)$ with a significant difference $(P<0.05)$ between these two figures. Likewise, there was a significant difference between the \% decrease in $\Delta \mathrm{LH}$ in responders and \% increase in $\Delta \mathrm{LH}$ in non-responders $(73.4 \pm 1.1 \%$ and $163.9 \pm 29.4 \%$, respectively). As in the case of basal $\mathrm{LH}$, testosterone levels tended to fall in responders with Brc in contrast to the increasing tendency in non-responders. No discernible change was recognized with basal and $\triangle F S H$. With Brc treatment, basal PRL was significantly decreased and $\triangle \mathrm{PRL}$ markedly decreased, both in responders and non-responders. Though there was no significant difference in the \% decrease in basal PRL in the two groups, the \% decrease in $\mathrm{PRL}$ in responders $(6.3 \pm 3.3 \%)$ was significantly smaller $(P<0.05)$ than that in non-responders $(21.4 \pm 4.6 \%)$.

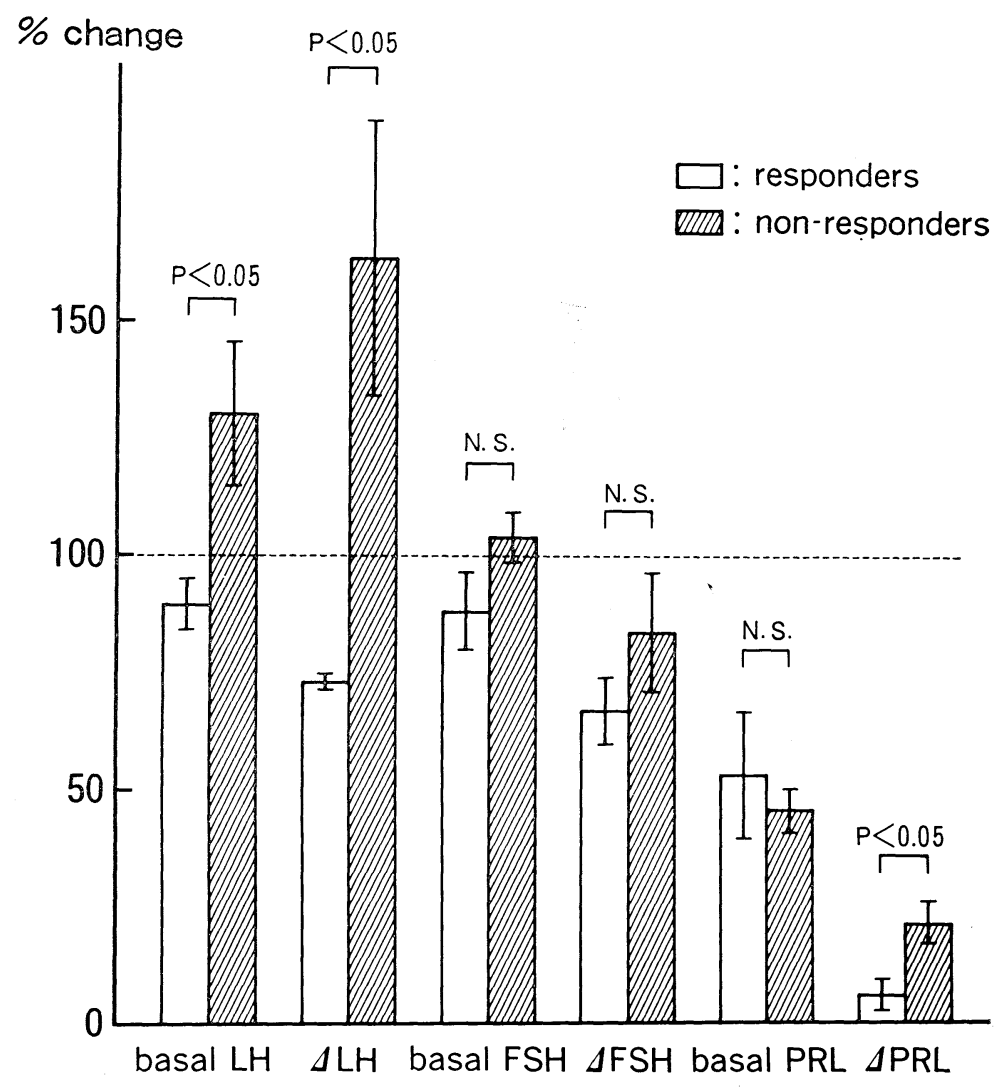

Fig. 1. Percent changes $( \pm$ SEM) in basal LH, $\triangle \mathrm{LH}$, basal FSH, $\triangle \mathrm{FSH}$, basal PRL and $\triangle \mathrm{PRL}$ after bromocriptine treatment in PCOS patients.

Percent changes signify comparison of post-treatment values with pretreatment values. $\Delta \mathrm{LH}$ and $\triangle F S H$ signify increases in LH and FSH after GnRH administration, respectively. $\angle \mathrm{PRL}$ signifies the increase in PRL after TRHadministration. 


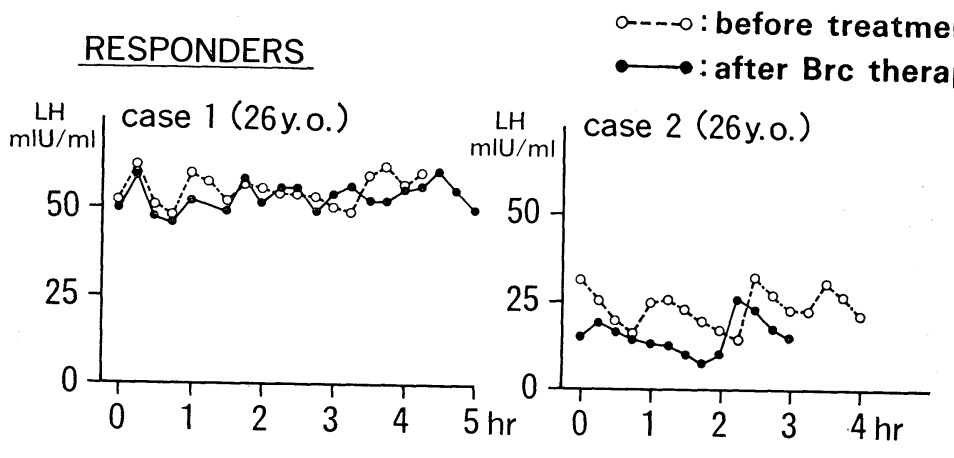

\section{NON-RESPONDERS}
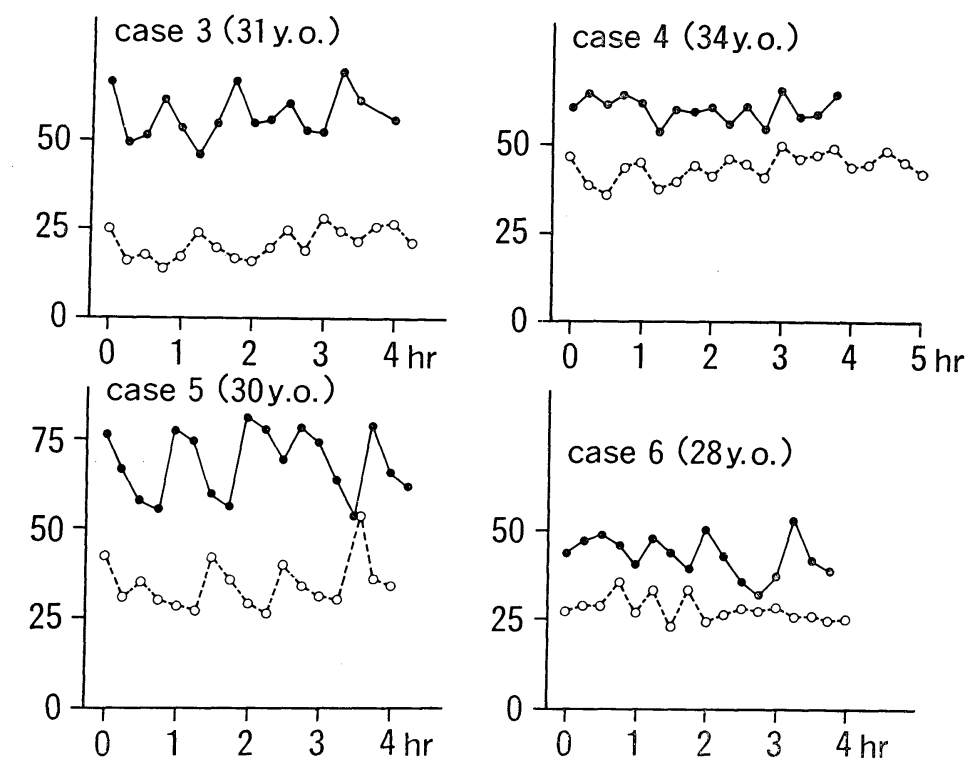

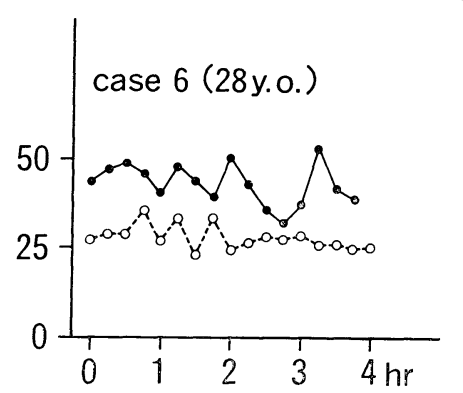

Fig. 2. Change in the pulsatile LH secretion pattern in PCOS patients before and after bromocriptine therapy.

Blood sampling was performed every $15 \mathrm{mi}$ nutes. In cases 1 and 2 ovulation was restored with bromocriptine therapy (responders). Cases 3 to 6 were unresponsive to bromocriptine therapy (non-responders).
The change in the pulsatile LH secretion pattern before and after Brc treatment is shown in Fig. 2. In responders, the baseline of LH secretion was almost unaffected (case 1) or slightly reduced (case 2) with the treatment, while the baseline and amplitude of $\mathrm{LH}$ secretion in non-responders even increased markedly.

\section{Discussion}

The present investigation unexpectedly demonstrated the existence of two subsets of clomiphene-resistant PCOS with different responses to Brc in terms of restoring ovulation and hormonal changes.

Brc, at the inception of its use for patients with PCOS, was welcome as a novel tool to restore ovulatory cycles (Spruce et al., 1984, Falaschi et al., 1986) in line with the concept of relative insufficiency of DA inhibition of $\mathrm{LH}$ secretion with this syndrome (Quigley et al., 1981, Cumming et al., 1984). Recently, this therapy has been criticized, because of the lack of significantly higher clinical effectiveness in PCOS patients than in the control subjects 
as shown by a large-scale randomized double blind study (Buvat et al., 1986). However, it should be carefully noticed that randomly selected patients in the whole spectrum of heterologous PCOS were included in that study. In our present study, though fundamental diagnostic criteria of menstrual disorder, LH hypersecretion and heperandrogenemia, were common to the previous study, ovulation was successfully induced with $\mathrm{Brc}$ in responders if a fraction of PCOS was selected by the additional criterion of clomiphene-resistency. Since clomiphene-resistence may signify a more severe or advanced state among overall cases of PCOS, this fact seems informative in the clinical sense that Brc can be a choice as a treatment to induce ovulation in these clomiphene-resistant PCOS patients before stepping up to $\mathrm{hMG}-\mathrm{hCG}$ treatment which has been shown widely to elicit severe ovarian hyperstimulation syndrome in PCOS (Raj et al., 1977, Wang et al., 1980).

Increased serum DHAS in non-responders before treatment was one of the conspicuous endocrine features in contrast to normal DHAS levels in responders. This observation might suggests the existence of a link to adrenal hyperfunction in these patients. Increased circulating levels of DHAS (Carter et al., 1977, Kandeel et al., 1978) without increased ASD (Vermeulen et al., 1977, Bassi et al., 1977) in hyperprolactinemic women has been well documented, and a positive correlation between PRL and DHAS has also been found (Jones et al., 1980). Though hyperprolactinemic PCOS was excluded from the present study, it was noteworthy that differences between percent changes in the increase in PRL ( $\triangle \mathrm{PRL})$ in response to TRH became significantly greater in nonresponders than in responders after a longterm period of Brc treatment, suggesting a relative lack of response to Brc of PRL secretion in the former.

Aggravation of $\mathrm{LH}$ hypersecretion in non-responders, but not in responders was another feature of hormonal changes induced by Brc treatment. Although a marked change was observed in pulsatile secretion pattern of $\mathrm{LH}$ after this treatment, the difference in $\mathrm{LH}$ secretion in responders and non-responders was apparent when comparing the percent changes in basal as well as $\Delta \mathrm{LH}$ in the two groups, both of which became significantly different after the treatment. This aggravating effect of Brc on LH secretion in non-responders is unexpected, when one considers the available data concerning dopaminergic mechanisms in controlling $\mathrm{LH}$ secretion that have been controversial (Rosen \& Lobo 1987), with the reports of inhibitory effects of DA and its agonist on $\mathrm{LH}$ secretion either in normal or hyperprolactinemic woman (Leblanc et al., 1976, Lachelin et al., 1977). Other reports deny such an inhibitory action in normal (Tolis et al., 1975, Polansky et al., 1976) and in hyperprolactinemic women (Evans et al., 1980).

There has been only one study that reported a differential action of DA and Brc on $\mathrm{LH}$ secretion (Martin et al., 1981), in which Brc was shown to slightly but definitely stimulate LH secretion, while DA lowered LH levels in normal women. The authors explained this discrepancy as a consequence of Brc binding to presynaptic receptors of multiple DA receptors in the hypothalamus. This, in turn, blocks the tonic inhibitory action of endogenous DA via postsynaptic receptors that is directly involved in $\mathrm{GnRH}$ secretion, as has recently been demonstrated in vitro in humans (Rasmussen et al., 1986).

In conclusion, the increase in circulating DHAS can serve as a clinical index to predict the efficiency of $\mathrm{Brc}$ for patients with clomiphene-resistant PCOS. In PCOS patients with increased serum DHAS, Brc is not only ineffective in ovulation induction but also has an aggravating effect on $\mathrm{LH}$ secretion. 


\section{Acknowledgements}

This work was supported in part by the Grants-in-Aid (\#61480350 and \#61570790) for Scientific Research from the Japanese Ministry of Education, Science and Culture. The authors are grateful to Sandoz Japan (Tokyo) for financial support and to Miss C. Tsuji and Miss M. Shimizu for technical assistance.

\section{References}

Aono, T., M. Miyazaki, A. Miyake, T. Kinugasa, K. Kurachi and K. Matsumoto (1977). Responses of serum gonadotrophins to $\mathrm{LH}^{-}$ releasing hormone and oestrogens in Japanese women with polycystic ovaries. Acta Endocrinol. (Copenh.) 85, 840-849.

Bassi, F., G. Giusti, L. Borsi, S. Cattaneo, P. Giannotti, G. Forti, M. Pazzagli, C. Vigiani and M. Serio (1977). Plasma androgens in women with hyperprolactinaemic amenorrhoea. Clin. Endocrinol. (Oxf.) 6, 5-10.

Buvat, J., M. Buvat-Herbaut, G. Marcolin, A. Racadot, J. C. Fourlinnie, R. Beuscart and P. Fossati (1986). A double blind controlled study of the hormonal and clinical effects of bromocriptine in the polycystic ovary syndrome. J. Clin. Endocrinol. Metab. 63, 119-124.

Carter, J. N., J. E. Tyson, G. L. Warne, A. S. McNeilly, C. Faiman and H. G. Friesen (1977). Adrenocortical function in hyperprolactinemic women. J. Clin. Endocrinol. Metab. 45, 973-980.

Coney, P. (1984). Polycystic ovarian disease: current concepts of pathophysiology and therapy. Fertil. Steril. 42, 667-682.

Cumming, D. C., R. L. Reid, M. E. Quigley, R. W. Rebar and S. S. C. Yen (1984). Evidence for decreased endogenous dopamine and opioid inhibitory influences on LH secretion in polycystic ovary syndrome. Clin. Endocrinol. (Oxf.) 20, 643-648.

Evans, W. S., A. D. Rogol, R. M. MacLeod and M. O. Thorner (1980). Dopaminergic mechanisms and luteinizing hormone secretion. I. Acute administration of dopamine agonist bromocriptine does not inhibit luteinizing hormone release in hyperprolactinemic women. J. Clin. Endocrinol. Metab. 50, 103-107.
Falaschi, P., A. Rocco and E. Del Pozo (1986). Inhibitory effect of bromocriptine treatment on luteinizing hormone secretion in polycystic ovary syndrome. J. Clin. Endocrinol. Metab. 62, 348-351.

Futterweit, W. (1984). Hypothesis and summary. In: Polycystic Ovarian Disease (W. Futterweit). Springer-Verlag, New York. pp. 163166.

Jones, D. L., H. S. Jacobs and V. H. T. James (1980). The relationship between plasma prolactin and dehydroepiandrosterone and dehydroepiandrosterone sulphate levels in patients with hyperprolactinaemia. In : Adrenal androgens (A. R. Genazzani, J. H. H. Thijssen and P. K. Siiteri ed.), Raven Press, New York, pp 83-87.

Kandeel, F. R., B. T. Rudd, W. R. Butt, R. Logan Edwards and D. R. London (1978). Androgen and cortisol responses to ACTH stimulation in women with hyperprolactinaemia. Clin. Endocrinol. (Oxf.) 9, 123-130.

Kokubu, T., M. Hisatomi, T. Ohuchi, H. Mori, A. Kambegawa, K. Furuya, K. Den, T. Yoshida and S. Takagi (1978). The simple methods for radioimmunoassay of unconjugated and sulfate conjugated dehydroepiandrosterone. Folia Endocrinol. Jap. 54, 117-130. (in Japanese).

Kurachi, K., M. Miyazaki, S. Mizutani and K. Matsumoto (1971). Plasma testosterone and urinary steroids in Japanese woman with polycystic ovaries. Acta Endocrinol. (Copenh.) 68, 293-302.

Lachelin, G. C. L., H. Leblanc and S. S. C. Yen (1977). The inhibitory effect of dopamine agonists on LH release in women. J. Clin. Endocrinol. Metab. 44, 728-732.

Lachelin, G. C. L., M. Barnett, B. R. Hopper, G. Brink and S. S. C. Yen (1979). Adrenal function in normal women and women with the polycystic ovary syndrome. J. Clin. Endocrinol. Metab. 49, 892-898.

Leblanc, H., G. C. L. Lachelin, S. Abu-Fadil and S. S. C. Yen (1976). Effects of dopamine infusion on pituitary hormone secretion in humans. J. Clin. Endocrinol. Metab. 43, 668674.

Lin, K. C., H. Okamura, M. Koshida and T. Mori (1986). Thyrotropin-releasing hormone and gonadotropin-releasing hormone test to predict effectiveness of bromocriptine therapy in infertile women. Fertil. Steril. 46, 846-851. 
Loughlin, T., S. Cunningham, A. Moore, M. Culliton, P. P. A. Smyth and T. J. McKenna (1986). Adrenal abnormalities in polycystic ovary syndrome. J. Clin. Endocrinol. Metab. 62, 142-147.

Martin W. H., A. D. Rogol, D. L. Kaiser and M. O. Thorner (1981). Dopaminergic mechanisms and luteinizing hormone (LH) secretion. II. Differential effects of dopamine and bromocriptine on LH release in normal women. $J$. Clin. Endocrinol. Metab. 52, 650-656.

Ohara, A., T. Mori, S. Taii, C. Ban and K. Narimoto (1987). Functional differentiation in steroidogenesis of two types of luteal cells isolated from mature human corpora lutea of menstrual cycle. J. Clin. Endocrinol. Metab. $65,1192-1200$.

Orsini, L. F., S. Venturoli, R. Lorusso, V. Pluchinotta, R. Paradisi and L. Bovicelli (1985). Ultrasonic findings in polycystic ovarian disease. Fertil. Steril. 43, 709-714.

Parisi, L., M. Tramonti, L. E. Derchi, S. Casciano, A. Zurli and P. Rocchi (1984). Polycystic ovarian disease: Ultrasonic evaluation and correlations with clinical and hormonal data. J. Clin. Ultrasound. 12, 2126.

Polansky, S., E. Muechler and S. Sorrentino Jr (1976). The effect of L-dopa and clomiphene citrate on peripheral plasma levels of luteinizing hormone-releasing factor. Obstet. Gynecol. $48,79-83$.

Quigley, M. E., J. S. Rakoff and S. S. C. Yen (1981). Increased luteinizing hormone sensitivity to dopamine inhibition in polycystic ovary syndrome. J. Clin. Endocrinol. Metab. 52, 231-234.

Raj, S. G., M. J. Berger, E. M. Grimes and M. L. Taymor (1977). The use of gonadotropins for the induction of ovulation in women with polycystic ovarian disease. Fertil. Steril. 28, 1280-1284.

Rasmussen, D. D., J. H. Liu, P. L. Wolf and S. S. C. Yen (1986). Gonadotropin-releasing hormone neurosecretion in the human hypothalamus: In vitro regulation by dopamine. J. Clin. Endocrinol. Metab. 62, 479-483.

Rebar. R., H. L. Judd, S. S. C. Yen, J. Rakoff, G. Vandenberg and F. Naftolin (1976). Characterization of the inappropriate ganadotropin secretion in polycystic ovary syndrome. $J$. Clin. Invest. 57, 1320-1329.

Rosen, G. F. and R. A. Lobo (1987). Further evidence against dopamine deficiency as the cause of inappropriate ganadotropin secretion in patients with polycystic ovary syndrome. J. Clin. Endocrinol. Metab. 65, 891-895.

Spruce, B. A., P. Kendall-Taylor, W. Dunlop, A. J. Anderson, M. J. Watson, D. B. Cook and C. Gray (1984). The effect of bromocriptine in the polycystic ovary syndrome. Clin. Endocrinol. (Oxf.) 20, 481-488.

Tolis, G., E. J. Pinter and H. G. Friesen (1975). The acute effect of 2-bromo- $\alpha$-ergocryptine (CB-154) on anterior pituitary hormones and free fatty acids in man. Int. J. Clin. Pharmacol. 12, 281-283.

Vermeulen, A, E. Suy and R. Rubens (1977). Effect of prolactin on plasma DHEA(S) levels. J. Clin. Endocrinol. Metab. 44, 1222-1225.

Wang, C. F. and C. Gemzell (1980). The use of human gonadotropins for the induction of ovulation in women with polycystic ovarian disease. Fertil. Steril. 33, 479-486.

Yen, S. S. C. (1980). The polycystic ovary syndrome. Clin. Endocrinol. (Oxf.) 12, 177208. 